The Institutional Repository IRUA is a subset of the Academic bibliography. As of 1991 the UA library produces an academic bibliography for the University of Antwerp (UA). IRUA contains scientific publications of UA researchers. If allowed the fulltext of the publications are made available.

This is a copy of an article published in the Journal of neurotrauma $\odot 2008$ [copyright Mary Ann Liebert, Inc.]; Journal of neurotrauma is available online at: http://online.liebertpub.com. 


\title{
Progression of Traumatic Intracerebral Hemorrhage: A Prospective Observational Study
}

\author{
RAJ K. NARAYAN, ${ }^{1}$ ANDREW I.R. MAAS, ${ }^{2}$ FRANCO SERVADEI, ${ }^{3}$ BRETT E. SKOLNICK, ${ }^{4}$ \\ MICHAEL N. TILLINGER, ${ }^{4}$ LAWRENCE F. MARSHALL, 5 \\ and THE TRAUMATIC INTRACEREBRAL HEMORRHAGE STUDY GROUP6
}

\begin{abstract}
Preliminary evidence has shown that intracerebral hemorrhages, either spontaneous (sICH) or traumatic (tICH) often expand over time. An association between hemorrhage expansion and clinical outcomes has been described for sICH. The intent of this prospective, observational study was to characterize the temporal profile of hemorrhage progression, as measured by serial computed tomography (CT) scanning, with the aim of better understanding the natural course of hemorrhage progression in tICH. There was also a desire to document the baseline adverse event (AE) profile in this patient group. An important motive for performing this study was to set the stage for subsequent studies that will examine the role of a new systemic hemostatic agent in tICH. Subjects were enrolled if they had tICH lesions of at least $2 \mathrm{~mL}$ on a baseline CT scan obtained within $6 \mathrm{~h}$ of a head injury. CT scans were repeated at 24 and $72 \mathrm{~h}$. Clinical outcomes and pre-defined AEs were documented. The data showed that $51 \%$ of the subjects demonstrated an increase in $\mathrm{IICH}$ volume, and that most of the increase occurred early. In addition, larger hematomas exhibited the greatest expansion. Thromboembolic complications were identified in $13 \%$ of subjects. This study demonstrates that $\mathrm{IICH}$ expansion between the baseline and 24-h CT scans occurred in approximately half of the subjects. The earlier after injury that the initial CT scan is obtained, the greater is the likelihood that the hematoma will expand on subsequent scans. The time frame during which hemorrhagic expansion occurs provides an opportunity for early intervention to limit a process with adverse prognostic implications.
\end{abstract}

Key words: cerebral contusions; deep vein thrombosis; intracerebral hemorrhage; thromboembolic complications; traumatic brain injury

\footnotetext{
${ }^{1}$ Department of Neurosurgery, University of Cincinnati, The Neuroscience Institute at University Hospital and The Mayfield Clinic, Cincinnati, Ohio.

${ }^{2}$ Department of Neurosurgery, University Hospital, Antwerp, Belgium.

${ }^{3}$ Neurosurgery-Neurotrauma Unit, University Hospital (Ospedale Maggiore), Parma, Italy.

${ }^{4}$ Novo Nordisk Inc., Princeton, New Jersey.

${ }^{5}$ Division of Neurosurgery, University of California, San Diego, California.

${ }^{6}$ The Traumatic Intracerebral Hemorrhage Study Group: U.S. Centers: N. Badjatia, Boston, MA; M. Cipolle, Allentown, PA; D. Duong, Los Angeles, CA; R. Dutton, Baltimore, MD; E. Dyer, Charlotte, NC; G. Fulda, Newark, DE; M. Grady, Philadelphia, PA; J. Hedges, Portland, OR; P. Hitchon, Iowa City, IA; S. Levy, Denver, CO; J. Linfoot, Houston, TX; M. McCarthy, Dayton, OH; J. Morris, Nashville, TN; J. Muizelaar, Sacramento, CA; R. Narayan, Cincinnati, OH; P. O’Neil, Brooklyn, NY; B. O’Neill, Salt Lake City, UT; K. O’Phelan, Honolulu, HI; J. Sava, Washington D.C; Z. Sifri, Newark, NJ; K. Smith, St. Louis, MO; N. Theodore, Phoenix, AZ; S. Timmons, Memphis, TN; D. Wang, Washington D.C; J. Ward, Richmond, VA; J. Wilberger, Pittsburgh, PA. Canadian Centers: M. Albert, Montreal; M. Cusimano, Toronto, ON; J. Fenwick, Vancouver, BC; A. Kaufmann, Winnipeg, MB; G. Pagliarello, Ottowa, ON; G. Poirier, Quebec City, QC; K. Reddy, Hamilton, ON; M. Chapman, Toronto, ON; B. Rowe, Edmonton, AB; D. Zygun, Calgary, AB.
} 


\section{NARAYAN ET AL.}

\section{INTRODUCTION}

$\mathbf{T}$ RAUMATIC BRAIN INJURY (TBI) is the most common cause of death and disability among young individuals (under the age of 40 years), and its incidence is increasing among the elderly (Kannus et al., 2001; Luukinen et al., 1999; Pace et al., 2006). TBI represents a wide spectrum of pathophysiological entities, including diffuse axonal injury with damage to white matter tracts, traumatic intracerebral hemorrhage ( $\mathrm{tICH})$, and extracerebral bleeding, with or without mass effect. The primary injury, inflicted at the moment of impact, initiates a complex sequence of events leading to secondary brain damage. This secondary damage may result from an expansion of hemorrhagic lesions, brain swelling, hypotension, hypoxia, hyperglycemia, pyrexia and the consequent activation of several biochemical cascades. Of the many potential secondary processes, hemorrhagic expansion is certainly one of the most important and devastating (Clifton et al., 1980) and the main causal factor in patients with head injury who "talk and die" (Reilly et al., 1975).

Several publications originating in the 1970s have demonstrated that ICH following TBI progresses over time (Brown et al., 1978; Diaz et al., 1979; Gudeman et al., 1979; Merino-deVillasante et al., 1976; Yamaki et al., 1990). All of these studies were retrospective reviews of data from a single center, with varying times of initial computed tomography (CT) scan, limited sample sizes, and semi-quantitative estimates for hemorrhage measurement. Since then, Servadei et al. (2000) demonstrated in a large, prospective, multicenter study that $20 \%$ of patients admitted with an initial diagnosis of diffuse injury, progressed to a mass lesion requiring surgical intervention.

The concept of lesion progression has been addressed in three recent papers (Chang et al., 2006; Chieregato et al., 2005; Oertel et al., 2002). Oertel et al. (2002) characterized the progression of hemorrhage in 142 TBI patients. Traumatic ICH was evident in approximately $49 \%$ of TBI patients who had CT scans performed within $2 \mathrm{~h}$ of injury and intracerebral hematomas expanded in $51 \%$ of patients with tICH. Chieregato et al. (2005) studied lesion evolution in a series of 141 patients admitted with a diagnosis of traumatic subarachnoid hemorrhage (SAH). Independent factors associated with significant CT progression were the amount of initial SAH, and both the presence of and the volume of brain hemorrhage upon admission. More specifically, only $3 \%$ of patients with no hemorrhage on admission had CT progression, as compared with $34 \%$ when there was a hematoma of less than $5 \mathrm{~mL}$ on admission and $60 \%$ when there was a larger hematoma.

Chang et al. (2006) quantified lesion size and further characterized the natural course of tICH. Four percent of the hemorrhagic lesions shrank, 58\% remained unchanged, and $38 \%$ grew. Initial baseline hematoma volume was found to be a strong predictor of hematoma expansion, with larger hematomas tending to increase in size, while smaller hematomas did not (for each $\mathrm{cm}^{3}$ of initial volume, the odds of growth increased by $11 \%$ ).

In patients with spontaneous intracerebral hemorrhage (sICH), hemorrhage expansion has been shown to play a significant role in a patient's clinical outcome (Broderick et al., 1993; Brott et al., 1997). A similar, although not as well documented, impact on outcome has been observed with hemorrhage growth in tICH. In a series of patients with closed head injury $(n=124)$ and Glasgow Coma Scale $(\mathrm{GCS})<8$, Clifton et al. (1980) showed that neurological deterioration occurred more frequently in trauma patients with intracerebral hematomas (14 of 31 subjects, $45 \%$ ) as compared with those with diffuse brain injury (10 of 69 subjects, $15 \%$ ). Of the subjects in coma who deteriorated, 19\% had large, delayed intracerebral hematomas.

Prior randomized clinical trials have focused primarily on neuroprotection. As no therapy has been identified (Doppenberg et al., 2004; Maas et al., 2007; Narayan et al., 2002), current strategies in treatment approaches to TBI have been merely supportive in nature. An alternative approach is to focus on methods of limiting hemorrhagic progression in TBI. The recent study of hemostatic agents in spontaneous ICH has rekindled an interest in the study of hematoma expansion in the tICH patient population with the aim of limiting this progression. This approach is particularly relevant, as patients with TBI have been reported to demonstrate a high rate of coagulopathy, which has been shown to be a significant independent predictor of hemorrhage progression and poor outcome (Engstrom et al., 2005; Stein et al., 1992, 2004). A potential disadvantage to the use of systemic hemostatic therapies is the risk of inducing thromboembolic complications. Prospective studies of hematoma expansion and the baseline incidence of thromboembolic events have not yet been evaluated in this population. In this study, we aimed to investigate the natural history of hematoma progression, with emphasis on hematoma size and expansion, in a systematic, quantitative, controlled clinical environment in patients with tICH. We also wanted to establish the baseline incidence of pre-specified adverse events (AEs) in this patient group.

\section{METHODS}

\section{Study Design}

This prospective, multi-center, observational study included the entire severity spectrum of closed head injury except for the most and least severe (GCS 4-14) with traumatic intracerebral hematomas that were not initially 
planned to have surgical evacuation. The study was conducted at 20 sites in the United States and Canada with approval from local Institutional Review Boards, as well as local and national ethics boards as applicable. Informed consent was obtained from the subject or a legally authorized representative prior to any study-related procedures. The active recruitment period was for 12 months. No investigational therapeutic agents were administered in this observational study. All subjects received standard care for TBI in accordance with guidelines published by the Brain Trauma Foundation (BTF), American Brain Injury Consortium (ABIC), and the American Association of Neurological Surgeons (AANS) (Bullock et al., 2000). The standard of care, at each study center, was evaluated by an independent Committee of Management Adherence (COMA) to ensure adherence to protocol and treatment guidelines. No treatments were withheld for any subject enrolled in this study.

Subjects were initially enrolled in the study if hemorrhage/contusion(s) of at least $5 \mathrm{~mL}$ were evident on baseline CT scans, obtained within $4 \mathrm{~h}$ of injury. After $13 \%$ of the subjects had been enrolled, the study was amended to improve recruitment by changing the minimum lesion volume from 5 to $2 \mathrm{~mL}$, the time from injury to baseline CT scan from 4 to $6 \mathrm{~h}$, and the baseline GCS to include scores of 4-14. Frequent examinations, including head CT scans at 24 and $72 \mathrm{~h}$ following injury, and other standard clinical assessments were performed during the first 5 days. Additional CT scans were performed at the discretion of the investigator. The study duration was 15 days with daily recording of AEs, with a special focus on thromboembolic complications. Routine ultrasound scanning of the lower extremities for deep vein thrombosis (DVT) was scheduled at $72 \mathrm{~h}$ post-injury. Clinical assessments included the GCS, the extended Glasgow Outcomes Scale (GOSE), and the Barthel Index (BI), evaluated at Day 15 or upon discharge, whichever came first.

It was originally anticipated that 100 subjects would be enrolled. However, the study was terminated by the sponsor after the enrollment of 63 subjects due to slow recruitment, and because it was believed that the number of recruited subjects with evaluable data was adequate to meet the study objectives.

\section{Study Population}

Subjects between the ages of 18 and 85 years who had clinical evidence of TBI (GCS 4-14), and tICH confirmed by CT scan (presence of hemorrhagic lesion(s) of $\geq 2 \mathrm{~mL}$ ) were enrolled in this study. Exclusion criteria included the presence of penetrating head injury or spinal cord injury, life expectancy of less than $8 \mathrm{~h}$ after hospital admission, and any planned surgical evacuation of intracerebral hematoma within $24 \mathrm{~h}$ after initial injury.
Furthermore, subjects with isolated subarachnoid hemorrhage, intraventricular hemorrhage, epidural or subdural hematomas or sICH, significant cardiovascular disease or hemodynamic instability, and pregnancy were excluded.

\section{Computed Tomography Image Analysis}

The initial head CT scan was performed immediately upon presentation (per standard clinical procedures) and no later than $6 \mathrm{~h}$ after the injury. The diagnosis of TBI was made on the basis of history, physical findings and radiological criteria. The initial evaluation of hematoma volume for inclusion was made by the investigator using the ABC/2 method (Kothari et al., 1996). CT scans were repeated at 24 and $72 \mathrm{~h}$ after injury. Additional CT scans were performed at the discretion of the investigator.

All data from the CT scans were transmitted in digital format to the Imaging Core Laboratory (Bio-Imaging Technologies, Inc., Newtown, PA). Computerized planimetric volumes of all intracranial hemorrhages were calculated. The contusions were compartmentalized where possible (e.g., hemorrhage and edema) using Analyze ${ }^{\mathrm{TM}}$ software (Mayo Clinic, Rochester, MN) with interactive display, processing, and measurement capabilities. Additional evidence of hemorrhage type (e.g., intraventricular, subdural, subarachnoid) was also recorded. All CT scans were evaluated in a temporal sequence by two independent neuroradiologists masked to subject and study center information. The data from these measurements were used for all subsequent analyses. If a subject underwent surgical evacuation of hematomas, the data obtained for the time points prior to surgery were included in the analyses.

\section{Safety Assessments}

All AEs, including specific pre-defined AEs, were reported for all subjects from admission until Day 15 or discharge from hospital, whichever occurred first. These critical events included brain death, DVT, pulmonary embolism (PE), myocardial infarction (MI; defined as chest pain, acute change in blood pressure and pulse, arrhythmias, signs of pump failure and/or ECG changes with ST elevations and increased troponin-I elevations), cerebral infarction (CI), vasospasm, and disseminated intravascular coagulation (DIC)/coagulopathy (based upon the definition from the International Society on Thrombosis and Haemostasis [ISTH]) (Carrick et al., 2005). An ultrasound screening of the lower extremities for DVT was mandated at $72 \mathrm{~h}$.

\section{Statistical Analysis}

As an observational study, no formal sample size calculations were performed. It was anticipated that enroll- 


\section{NARAYAN ET AL.}

ment of 50-100 subjects would be required to permit meaningful analysis, based on the assumption that approximately $50 \%$ of the subjects would experience hematoma expansion. Descriptive statistics were used to describe the subject characteristics. In this study, hematoma progression was assessed by evaluating both hemorrhage volume changes as well as volume increases. The term "volume change" included both increases as well as decreases in hematoma size, while "volume increases" included only expansions in hematoma size. Changes in hemorrhage volume from baseline to 24 and $72 \mathrm{~h}$ post-injury were analyzed by a generalized linear mixed model. For analyses evaluating tICH volume increases the distribution was truncated, since subjects with decreases in tICH from baseline were excluded, resulting in invalid variance estimates and meaningless $p$-values. Logistic regression analysis was performed to investigate the relationship between CT characteristics, hematoma expansion, and the BI. The $p$-values were calculated based on a linear mixed model with two repeated factors: reader and visit.

\section{RESULTS}

\section{Subject Recruitment}

A total of 3,232 subjects diagnosed with TBI and having an initial CT scan were screened for entry into the study. However, fewer than 2\% (63 subjects) of those screened were determined to be eligible for enrollment. The high screen exclusion rate was due to the restrictive enrollment criteria and the stringent initial tICH volume in combination with the large number of TBI patients with baseline CT scans without evidence of hemorrhage. Other major reasons for screening exclusions included lesion volumes of $<2 \mathrm{~mL}(30 \%)$, GCS outside the prescribed range $(23 \%)$, presentation outside the protocol mandated time window ( $9 \%)$, age outside required range $(9 \%)$, penetrating head injury and isolated extra-axial hemorrhage $(6 \%)$, inability to obtain informed consent within the appropriate time window (4\%), and spinal cord injuries (3\%).

Sixty subjects were analyzed as part of the safety population. For four subjects, the baseline CT scans were not of adequate quality to accurately assess tICH volume evolution. Therefore, 56 subjects had evaluable data for hematoma progression and comprised the observed population. The term "observed population" is used in lieu of "intent-to-treat" as this was an observational study.

\section{Baseline Characteristics}

The baseline characteristics of the subject population are summarized in Table 1. Subjects enrolled were pre- dominantly male Caucasians with a mean age of 43.8 years (range, 18-84 years). The median GCS was 8.0, and motor vehicular accidents were the cause of $47 \%$ of the TBIs, with falls being the second most frequent cause. Ten subjects $(17 \%)$ were identified as being coagulopathic at baseline (defined as platelets $\leq 100,000, \mathrm{PT}>$ $14 \mathrm{sec}$, PTT $>45 \mathrm{sec}$ or INR $\geq 1.2$ ). AANS/BTF/ABIC treatment guidelines were adhered to in 45 subjects (75\%). The management of the remaining $25 \%$ was not entirely consistent with the treatment guidelines. The median baseline GCS for this latter group was 7.0 compared to 9.0 for those subjects who maintained treatment targets. GCS assessment between these two groups was different $(p<0.02)$ at all time points. At discharge/D15 the final GCS was 7.0 for those who were unable to meet treatment guidelines and 14.0 for those who met treatment targets.

On central review of the baseline CT scan, 91\% $(n=$ $51)$ of the enrolled subjects were confirmed as having $\mathrm{tICH}$ of $\geq 2 \mathrm{~mL}$. The average volume of $\mathrm{tICH}$ lesions at

Table 1. Subject Demographics and Baseline Characteristics

\begin{tabular}{lc}
\hline Parameter & $\mathrm{n}=56$ \\
\hline Age, years & \\
Mean (SD) & $43.8(19.6)$ \\
Median & 42.5 \\
Range & $18-84$ \\
Male gender, $n(\%)$ & $42(75)$ \\
Caucasian ethnicity, $n(\%)$ & $46(82)$ \\
Baseline Glasgow Coma Scale & \\
Median & 8 \\
Range & 4 to 15 \\
Marshall Classification, $n(\%)$ & 0 \\
Grade 1 & $32(56)$ \\
Grade 2 & $10(18)$ \\
Grade 3 & $6(11)$ \\
Grade 4 & 0 \\
Grade 5 & $9(16)$ \\
Grade 6 & \\
Causes of injury, $\%$ & 47 \\
Motor vehicular accidents & 35 \\
Falls &
\end{tabular}

Grade 1: No intracranial pathology visible on CT scan.

Grade 2: Lesions and/or midline shift $\leq 5 \mathrm{~mm}$ with open basal cisterns. No high or mixed density lesion $>25 \mathrm{~mm}$.

Grade 3: Midline shift $\leq 5 \mathrm{~mm}$ with cisterns compressed or absent. No high or mixed density lesions $>25 \mathrm{~mm}$.

Grade 4: Midline shift $>5 \mathrm{~mm}$. No high or mixed density lesions $>25 \mathrm{~mm}$.

Grade 5: Any surgically evacuated mass.

Grade 6: High or mixed density lesion $>25 \mathrm{~mm}$, not surgically evacuated. 


\section{HEMORRHAGE PROGRESSION IN TRAUMATIC BRAIN INJURY}

baseline was $8.8 \mathrm{~mL}$. Fourteen subjects were enrolled with an initial lesion volume of $2-5 \mathrm{~mL}, 34$ subjects with lesion volumes greater than or equal to $5 \mathrm{~mL}$, and eight subjects with baseline lesion volumes smaller than $2 \mathrm{~mL}$ (the initial estimations of $\mathrm{tICH}$ volume made by the investigators using the $\mathrm{ABC} / 2$ method was greater than $2 \mathrm{~mL}$ ). Overall, there was no difference in the measurement of tICH volume size between the two independent readers of the CT scans. The intra-class correlation coefficient for the determination of $\mathrm{tICH}$ volume between the two independent readers was 0.9613. Concurrent hematoma/hemorrhage types included subarachnoid (77\%), subdural (57\%), intraventricular $(23 \%)$, epidural $(12 \%)$, cerebellar contusion $(9 \%)$, and brainstem contusion (7\%). Of these subjects, only $5 \%$ had epidural and $3 \%$ had subdural evacuations during the study period.

\section{Progression of Traumatic Intracerebral Hemorrhage}

Table 2 summarizes tICH volume change and increase over 72 h. Volume change was defined as any increase or decrease in tICH volume, while volume increase described only tICH lesion expansion. When compared to baseline volumes, the mean change in tICH lesion volumes at 24 and $72 \mathrm{~h}$ were 3.6 and $2.7 \mathrm{~mL}$, respectively. These volume changes from baseline were statistically significant $(p=0.004$ and $p=0.018)$. The subsequent change in volume between 24 and $72 \mathrm{~h}(-0.82 \mathrm{~mL}, p=$ 0.28 ), however, indicated that the largest volume change occurred within the first $24 \mathrm{~h}$, with little further change thereafter.

Hematoma volume increased $7.3 \mathrm{~mL}$ between baseline and $24 \mathrm{~h}$ in 26 of the 51 (51\%) subjects (Table 2). At 72 $\mathrm{h}$, the $\mathrm{tICH}$ volume increase from baseline was $5.8 \mathrm{~mL}$ $(n=24)$, demonstrating that the largest $\mathrm{tICH}$ volume in- creases occurred within the first $24 \mathrm{~h}$. The data also indicated that hematoma expansion was observed more frequently in subjects in whom the initial baseline CT scan was performed early (Fig. 1). If the initial CT scan was conducted more than $3.5 \mathrm{~h}$ following injury then the percentage of subjects with measurable changes in hematoma volume was greatly reduced.

In this study, intermediate CT scans (conducted between the baseline and the 24-h CT scan) were obtained at the discretion of the physician for the subset of 25 $(50 \%)$ subjects. The presumption is that these additional CT scans were undertaken because of neurological deterioration. This subset of subjects provided an opportunity to evaluate lesion volumes at a time point earlier than the protocol-mandated 24-h CT scan. The mean interval between injury and the intermediate CT scan was $9.1 \pm 3.5$ $\mathrm{h}$, with the largest proportion of subjects having intermediate scans conducted at 6-9 h (Fig. 2). The mean volume change from baseline to the intermediate scan was $5.7 \mathrm{~mL}(p=0.0003$; Table 3$)$. The difference in mean tICH volume between the intermediate scan and $24 \mathrm{~h}$ was $0.3 \mathrm{~mL}$, indicating that the maximal change in $\mathrm{tCCH}$ volume had occurred by the intermediate scan. More rapid CT following injury ( $\leq 3.5 \mathrm{~h}$ compared to $>3.5 \mathrm{~h}$ ) resulted in greater proportion of patients with hematoma expansion measured at $24 \mathrm{~h}(57 \%$ vs. $28 \%)$, which was even more striking ( $80 \%$ vs. $20 \%$ ) when an intermediate CT was performed (Fig. 3).

Seventeen of the 25 subjects with an intermediate scan $(68 \%)$ demonstrated a mean increase in tICH volume of $9.3 \mathrm{~mL}$ (Table 3 ). The mean difference in volume increase between the intermediate time point and $24 \mathrm{~h}$ was only $0.2 \mathrm{~mL}$, indicating, similar to the measurements for tICH volume change, the maximal increase in volume had occurred by the intermediate scan (Table 3 ). For the $47 \%$ of subjects who did not have

Table 2. Traumatic ICH Volume Progression: Baseline, 24 and 72 H

\begin{tabular}{|c|c|c|c|}
\hline & Baseline, $\mathrm{n}=56^{\mathrm{a}}$ & 24 hours, $\mathrm{n}=51^{\mathrm{a}}$ & 72 hours, $\mathrm{n}=45^{\mathrm{a}}$ \\
\hline \multicolumn{4}{|l|}{ Subjects with volume change ${ }^{b}$ from baseline } \\
\hline$n(\%)$ & & $51(100)$ & $45(100)$ \\
\hline tICH volume, mL (SE) & $8.8(1.3)$ & $12.4(1.7)$ & $11.5(1.4)$ \\
\hline Mean volume change (SE), mL & & $3.6(1.21), p=0.004$ & $2.7(1.14), p=0.018$ \\
\hline \multicolumn{4}{|l|}{ Subjects with volume increase ${ }^{c}$ from baseline } \\
\hline$n(\%)$ & & $26(51)$ & $24(53)$ \\
\hline tICH volume, mL (SE) & $7.39(0.97)$ & $14.7(2.02)$ & $13.2(1.25)$ \\
\hline Mean volume change (SE), mL $[95 \% \mathrm{Cl}]$ & & $7.3(1.53)[4.29,10.38]$ & $5.8(1.25)[3.31,8.29$ \\
\hline
\end{tabular}

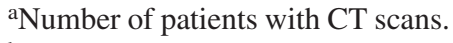

${ }^{\mathrm{b}} \mathrm{Change}$ from baseline includes increases as well as decreases in $\mathrm{tICH}$ volume from baseline.

'Increase in volumes describes expansion of tICH lesion size.

$p$-values were calculated based on a linear mixed model with two repeated factors: reader and visit. 


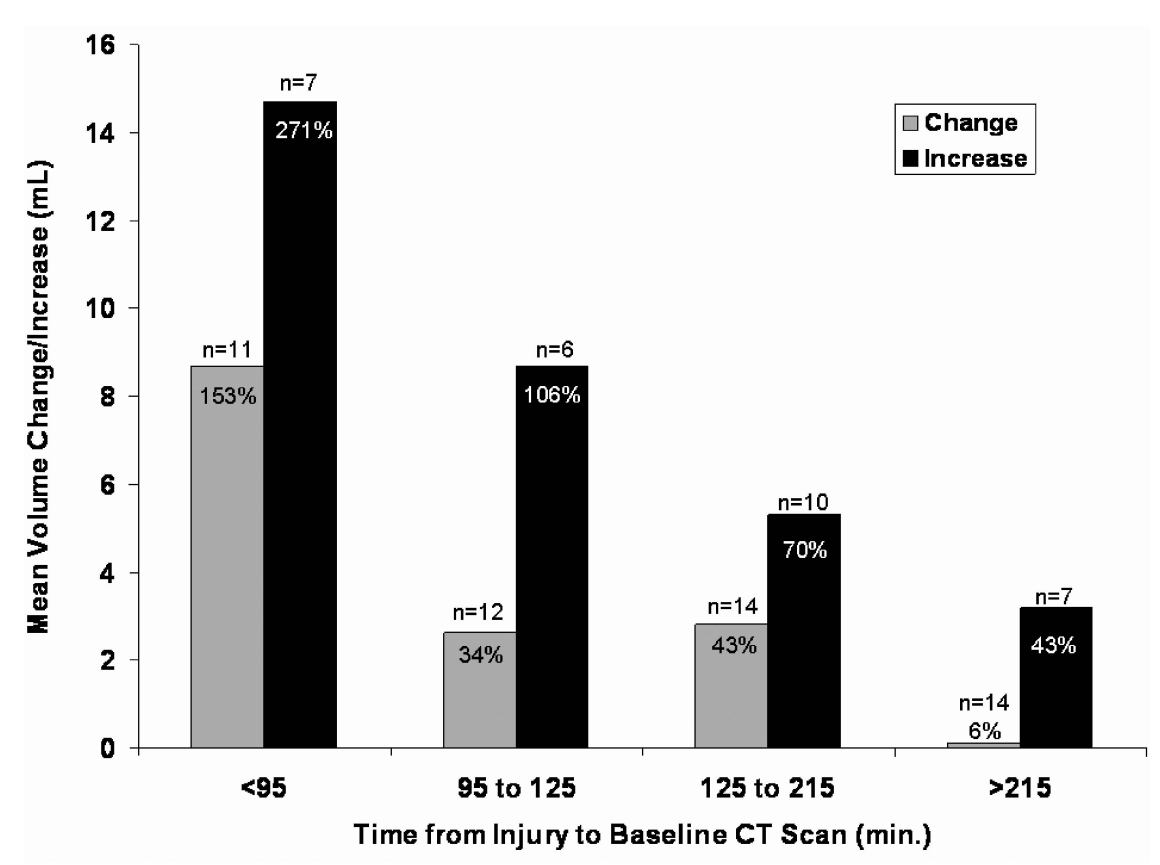

FIG. 1. Mean traumatic intracerebral hemorrhage (tICH) volume change and increase at $24 \mathrm{~h}$, by time from injury to baseline computed tomography (CT) scan. n represents the number of subjects within each time window, and the percentages describe the mean tICH volume increase or change within the specified time window. As the time interval between injury and baseline $\mathrm{CT}$ scan increases, the percent $\mathrm{tICH}$ volume increase/change from baseline to $24 \mathrm{~h}$ decreases.

an intermediate scan, the mean volume increase between baseline and $24 \mathrm{~h}$ was $6 \mathrm{~mL}$.

Five subjects had six surgeries for intraparenchymal hematoma evacuation, all of whom had baseline tICH volumes greater than $5 \mathrm{~mL}$. Two of the surgeries occurred less than $8 \mathrm{~h}$ after injury, two between $8-24 \mathrm{~h}$ after injury, one between 24-72 h after injury, and one almost 6 days after injury.

\section{Baseline tICH Lesion Volume Progression}

Since previous reports have demonstrated that baseline hematoma volume is a strong predictor of hematoma increase (Chang et al., 2006; Chieregato et al., 2005), we investigated whether an association existed between the initial lesion volume at admission and lesion progression. Baseline lesion volumes were assigned to four groups ( $<2 \mathrm{~mL} ; \geq 2$ to $<5$; $\geq 5$ to $<11 \mathrm{~mL}$; and $\geq 11 \mathrm{~mL}$ ). Subjects with baseline hematoma volumes of $<2 \mathrm{~mL}$ were not excluded as this observational study also functioned as a feasibility study to evaluate the possibility of performing central CT scan reads, as well as to assess the underlying AE rate in this patient population. The largest mean volume increase $(14.5 \mathrm{~mL})$ over $24 \mathrm{~h}$ was observed in the 5-11 mL group (Table 4). Overall, there appeared to be a trend towards larger lesions $(5-11 \mathrm{~mL})$ demonstrating larger increases in hemorrhage volume, although the $\geq 11 \mathrm{~mL}$ group demonstrated less increase.

\section{Functional Outcomes}

There was no apparent association between baseline GCS and the presence of hematoma, hematoma size at baseline, nor expansion in lesion size.

The median extended GOSE for the 56 subjects with Day 15/Discharge data was 3.0. At Day 15/Discharge, $52 \%(29 / 56)$ of subjects had scores of 3-4 (i.e., lower to upper severe disability), limiting the possibility of analysis of the association between GOSE and lesion progression.

The BI permits a more sensitive analysis of subjects with lower GOSE. The mean and median BI for the 54 subjects with data at Day 15/Discharge were 34.5 \pm 10.0 and 41.1, respectively. In general there was a trend toward subjects with smaller baseline lesions having more favorable functional outcomes. However this trend was not statistically significant $(p=0.60)$ and given the relatively small sample size in this study no correlation with outcome could be reasonably expected. Regression analyses indicated that baseline lesion volume, change in lesion volume from baseline to $24 \mathrm{~h}$, age, and time to first CT scan were not predictive of BI at Day 15/Discharge.

An assessment of hematoma expansion and mortality indicated that of the 35 subjects with hematoma expansion, seven subjects $(20 \%)$ died. Of the remaining 25 subjects without hematoma expansion, only one subject (4\%) 


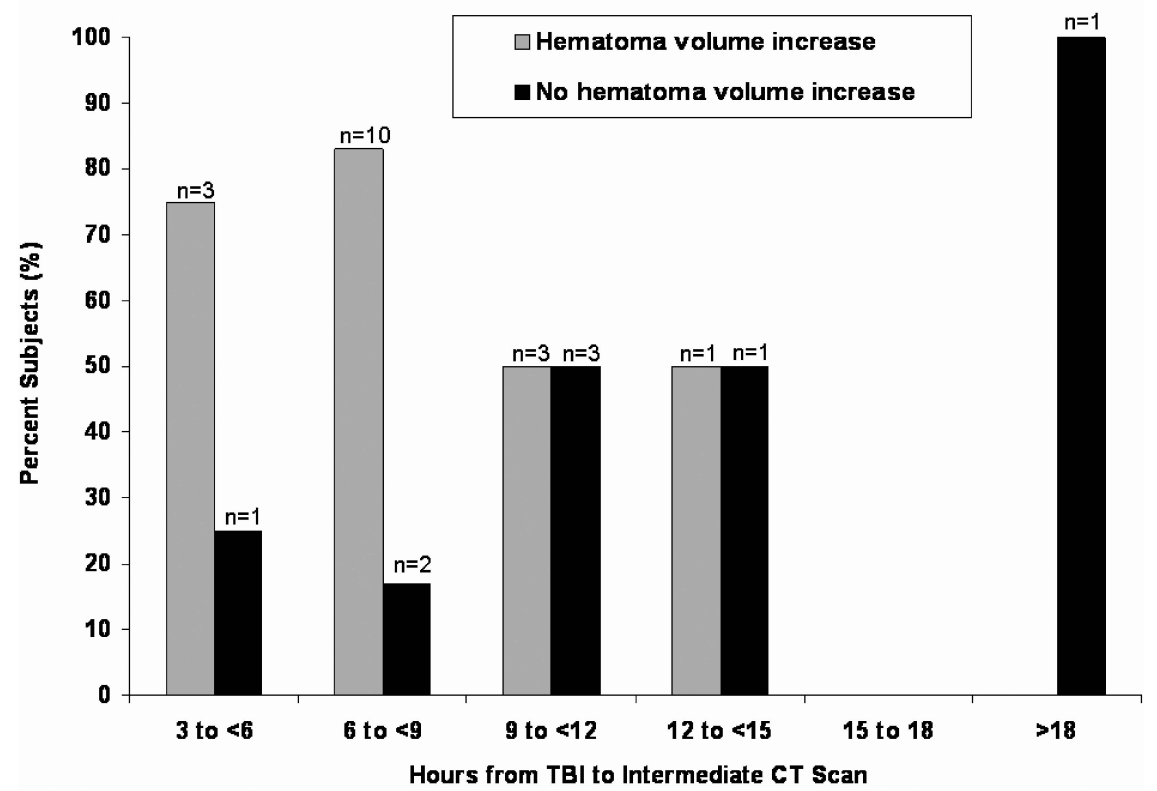

FIG. 2. Percentage of subjects with intermediate computed tomography (CT) scan demonstrating traumatic intracerebral hemorrhage (tICH) volume increase, by time from injury to intermediate $\mathrm{CT}$. $\mathrm{n}$ represents the number of subjects within the group. As the time interval between injury and the intermediate scan increases, the proportion of subjects demonstrating tICH volume increase decreases.

died. Subjects that died had a baseline tICH volume of $18.9 \mathrm{~mL}$, with a mean tICH volume change of $8.15 \mathrm{~mL}$ and $11.9 \mathrm{~mL}$ increase in tICH volume.

\section{Adverse Events}

Forty-eight of the $60(80 \%)$ subjects reported 256 AEs, of which 15 were serious in nature (Table 5). There were eight events that had been pre-specified to be of special interest with possible thromboembolic etiology. These included one event each of CI, PE, DIC, and elevated troponin-I level. Four DVTs were reported, two of which were clinically asymptomatic, detected by ultrasound of the lower extremity, and classified only as AEs.
The mortality rate at Day 15/Discharge was 8/60 (13\%). The most common causes of death (5/8 subjects) were related to brain injury, and included brain death, brain herniation, and hemorrhage expansion, All of these subjects demonstrated an increase in $\mathrm{tICH}$, and four of these subjects died following the intermediate $\mathrm{CT}$ scan but before the 24-h scan. DIC, CI, and cardiorespiratory arrest were the reported causes of death in the other 3 subjects.

\section{DISCUSSION}

The current study is the first multicenter, prospective study, with high-quality, source-verified data, as well as

Table 3. Traumatic ICH Volume Progression for Subjects with Intermediate Scans

\begin{tabular}{|c|c|c|c|}
\hline & Baseline, $\mathrm{n}=56^{\mathrm{a}}$ & Intermediate, $\mathrm{n}=25^{\mathrm{a}}$ & $24 h, \mathrm{n}=51^{\mathrm{a}}$ \\
\hline Subjects with volume change ${ }^{\mathrm{b}}, n(\%)$ & & $25(100)$ & $21(41)$ \\
\hline Mean volume (SE), $\mathrm{mL}$ & $8.11(1.02)$ & $13.82(1.77)$ & $13.53(1.87)$ \\
\hline Mean volume change (SE), mL & & $5.72(1.51), p=0.0003$ & $5.43(1.53), p=0.0007$ \\
\hline Subjects with volume increase ${ }^{\mathrm{c}}, n(\%)$ & & $17(68)$ & $12(57)$ \\
\hline Mean volume (SE), mL & $8.2(1.20)$ & $16.9(2.01)$ & $17.1(2.16)$ \\
\hline Mean volume change (SE), mL $[95 \% \mathrm{Cl}]$ & & $9.27(1.49)[6.18,12.36]$ & $9.96(1.82)[6.20,13.73]$ \\
\hline
\end{tabular}

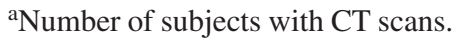

${ }^{\mathrm{b}}$ Change from baseline includes increases as well as decreases in tICH volume from baseline.

' Increase in volumes describes positive growth of tICH lesions.

$p$-values were calculated based on a linear mixed model with two repeated factors: reader and visit. 


\section{NARAYAN ET AL.}

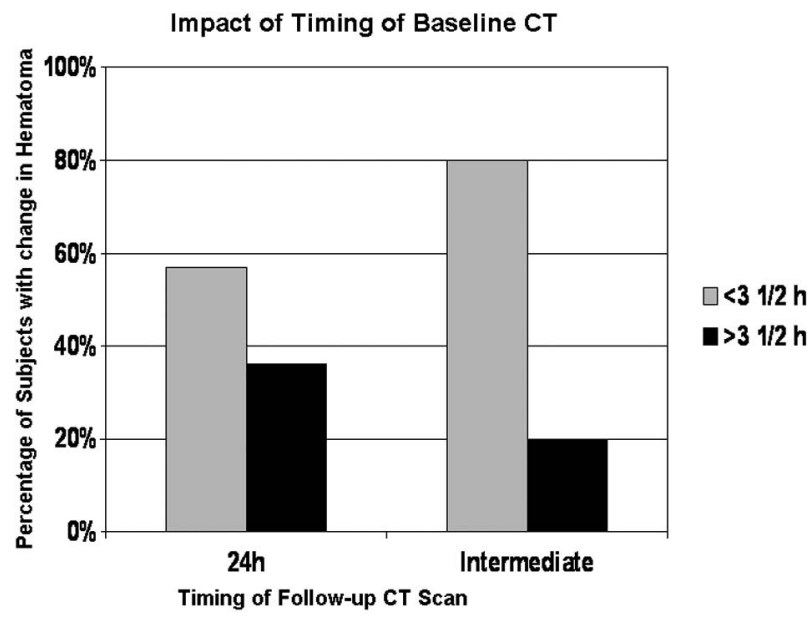

FIG. 3. Impact of timing of baseline computed tomography (CT) on follow-up CT scan for change in hematoma. In the patients scanned at $24 \mathrm{~h}$ (as per protocol), patients with an early initial CT (less than $3.5 \mathrm{~h}$ post-injury) showed hematoma expansion of $57 \%$ as compared $36 \%$ when their initial CT was after $3.5 \mathrm{~h}$ post-injury. This difference is even more evident in patients who underwent a second CT scan for clinical indications before the planned 24-h study (Intermediate CT-Scan Group). In this sub-set of $80 \%$ of patients, those scanned within $3.5 \mathrm{~h}$ of injury had hematoma expansion, compared to only $20 \%$ if they were scanned later than $3.5 \mathrm{~h}$ post-injury.

standardized CT collection and centralized CT analysis. The goals of the study were to describe the natural history of tICH and to investigate the incidence of thromboembolic complications in TBI subjects. In this study, it was demonstrated that tICH expansion occurred in 51\% of the subjects enrolled, a finding consistent with previous retrospective single-center reports (Chang et al., 2006; Oertel et al., 2002). Perhaps more importantly, this study provided evidence that hemorrhage progression primarily occurs within the first $24 \mathrm{~h}$, suggesting that any agent designed to limit hematoma expansion should be administered as early as possible following injury.
This study provided an opportunity for the analysis of additional physician-directed CT scans conducted prior to the protocol-mandated 24-h CT scan. These scans were presumably obtained because of clinical deterioration and therefore may represent a biased subset. The data indicated that these subjects demonstrated a greater likelihood of hemorrhage expansion as well as a larger volume increase, and that the majority of the volume increase occurred within $9 \mathrm{~h}$ from injury. For these subjects an association between possible clinical deterioration and lesion progression was also observed.

Many existing guidelines (Lobato et al., 1997; Procaccio et al., 2000) for the treatment of TBI already contain recommendations for repeat CT scans to be conducted within $24 \mathrm{~h}$. Our data suggest that in addition to routine clinical assessments, there may be potential value in conducting these CT scans even earlier in the course of treatment.

The results of this study also confirmed previous observations that while small tICH lesions $(<2 \mathrm{~mL})$ may expand, larger initial baseline lesions tend to have substantially greater hematoma increase, with a greater likelihood of clinical impact. Although, a consistent definition of lesion progression has not been established in TBI, lesion enlargements of $\leq 2 \mathrm{~mL}$ may not be relevant to the subject's clinical outcome. Traumatic ICH volume as reflected in increases of $25 \%$ (Oertel et al., 2002) and of $50 \%$ (Chieregato et al., 2005) in at least one dimension of the tICH have been considered significant. Mean volume increase of more than $5 \mathrm{~mL}$ in $24 \mathrm{~h}$ is probably relevant to patient outcomes and management and has been identified as a risk factor for late surgery (Chang et al., 2006). In this study, 29\% (15/51) of subjects demonstrated a tICH volume increase of greater than $5 \mathrm{~mL}$ between the baseline and the 24-h CT scan.

Previous studies have demonstrated that hematoma expansion in subjects with spontaneous ICH were associated with greater clinical morbidity, as determined by outcome scales (e.g., modified Rankin Scale [mRS], Oxford Handicap Scales) (Brott et al., 1997; Mayer et al., 2005).

Table 4. Traumatic ICH Volume Progression: Baseline, and 24 h, by Baseline Hematoma Size

\begin{tabular}{lcccc}
\hline & \multicolumn{4}{c}{ Baseline traumatic ICH volume $(\mathrm{mL})$} \\
\cline { 2 - 5 } & $<2, \mathrm{n}=8$ & $\geq 2$ to $<5, \mathrm{n}=14$ & $\geq 5$ to $<11, \mathrm{n}=19$ & $\geq 11, \mathrm{n}=15$ \\
\hline Subjects with tICH volume change, $n(\%)$ & $6(75)$ & $14(100)$ & $17(89)$ & $14(93)$ \\
Mean volume change (SD), $\mathrm{mL}$ & $0.5(1.3)$ & $3.1(4.9)$ & $4.3(14.5)$ & $3.5(9.5)$ \\
Subjects with tICH volume increase, $n(\%)$ & $2(25)$ & $9(64)$ & $7(37)$ & $8(53)$ \\
Mean volume increase (SD), $\mathrm{mL}$ & $1.9(1.32)$ & $5.3(4.72)$ & $14.5(18.72)$ & $10.2(6.52)$ \\
\hline
\end{tabular}

One subject in the $<2 \mathrm{~mL}$ group, two subjects in the $\geq 2$ to $<5 \mathrm{~mL}$ group, and one subject in the $\geq 11 \mathrm{~mL}$ group were missing 24 CT scans. 
Table 5. Serious Adverse Events and Predefined Critical Events with Potentially Thromboembolic Etiology

\begin{tabular}{|c|c|c|}
\hline & \multicolumn{2}{|c|}{ Total } \\
\hline & $\mathrm{n}(\%)$ & $\mathrm{E}(\%)$ \\
\hline No. of subjects & 60 & \\
\hline No. of AEs & $48(80)$ & 256 \\
\hline No. of SAEs & $12(20)$ & $15(6)$ \\
\hline No. of deaths & $8(13)$ & \\
\hline No. of predefined critical AAEs & $8(13)$ & $8(3)$ \\
\hline DIC & $1(2)$ & $1(<1)$ \\
\hline $\begin{array}{l}\text { Cerebral infarction/ } \\
\text { cerebral hemorrhage }\end{array}$ & $1(2)$ & $1(<1)$ \\
\hline Pulmonary embolism & $1(2)$ & $1(<1)$ \\
\hline Deep venous thrombosis ${ }^{a}$ & $4(7)$ & $4(2)$ \\
\hline Troponin-I elevated & $1(2)$ & $1(<1)$ \\
\hline
\end{tabular}

aTwo cases were described as serious, and two cases were described as non-serious.

n, number of subjects; E, number of events.

In the setting of TBI, Stein et al. (1993) showed that patients $(n=149)$ with delayed brain injury (hematoma progression or new hematoma) had a higher mortality, a slower recovery and a worse outcome. Patients with CT evolution have a statistically significant increase in the risk of unfavorable outcomes, as reported by a dichotomized GOS at 6 months from injury (Fainardi et al., 2004; Lobato et al., 1997; Servadei et al., 2000). It therefore seems similar to sICH in that a relationship between tICH evolution and long-term poor outcomes may exist, but studies with a sample size adequately powered to demonstrate have been difficult to perform.

We observed an association between possible clinical deterioration and lesion progression for subjects with tICH who had intermediate CT scans, providing some evidence to suggest the deleterious effect of tICH expansion in TBI, and arguing in favor of limiting lesion expansion. Based on this information, future trials investigating the impact of agents aimed at limiting expansion of tICH, may focus on $\mathrm{CT}$ measurements as an early mechanistic endpoint. The use of planimetric volume measurements as an accurate and reliable tool has been previously demonstrated (Zimmerman et al., 2006).

The use of such volumetric measurements would be of particular importance if a clear relationship can be established between hemorrhage progression and clinical outcome as it has been for spontaneous ICH (Broderick et al., 1993; Mayer et al., 2005).

Injury to the central nervous system and the associated complications of immobilization and altered levels of consciousness (due to sedation or injury) along with the high brain tissue factor release, secondary to injury, are significant risk factors for the development of arterial and venous thrombotic complications. Therefore, TBI patients may be at greater risk for thromboembolic events as compared with polytrauma or ICU subjects (Geerts et al., 2004). Limited data exist to describe the incidence of thromboembolic AEs for patients with TBI. For example, lower extremity ultrasound screens to monitor and detect DVTs have rarely been implemented in a TBI clinical protocol (Kurtoglu et al., 2004). In our study, the frequency and nature of AEs were carefully monitored. A total of four DVTs and one PE were observed for the 60 subjects who entered the trial. These data indicate that thromboembolic complications are not uncommon in TBI and careful monitoring for such thromboembolic complications is recommended in any future studies. These results provide a useful baseline against which future therapeutic trials can be compared.

Several limitations of the present study should be acknowledged. Only a highly selected subset of the TBI population was evaluated. This was done in an attempt to focus the highly diverse TBI patient group to evaluate a population of TBI patients that would be representative of a general trauma population but focused enough to identify a target population that could potentially benefit from therapeutic intervention. Therefore, individuals that demonstrated an initial hemorrhage and who presented shortly after injury were enrolled.

We did not report the development or progression of other components of the traumatic hemorrhagic lesions such as epidural hematoma, acute subdural hematoma, subarachnoid hemorrhage, or intraventricular hemorrhage. In order to avoid the difficulties in accounting for the effect of surgery on hematoma volumes and clinical outcome, it was decided by the Steering Committee, while planning the study, to exclude these lesion types from the study analyses. In addition, we did not investigate the development of new lesions in subjects for whom the initial CT did not show any intraparenchymal hemorrhage component; nor could we identify predictors of tICH progression. In addition, a confounding factor in analyzing lesion progression was the variability in the time interval between injury and baseline CT scan $(0.7-8.67 \mathrm{~h})$. However, greater standardization is not possible in clinical practice.

In this trial, the primary focus was on hemorrhage progression and immediate AE profiles. The outcome scores (GOSE and BI) were assessed at Day 15 or discharge and not at the conventional time point of 6 months. To date, most of the emphasis in TBI research has been on neuroprotection for long-term functional improvement (Narayan et al., 2002). Therefore, most of the clinical assessment tools in TBI have focused on long-term outcomes using the GOS or GOSE (Narayan et al., 1981, 


\section{NARAYAN ET AL.}

1982). The current TBI outcomes measures were not designed to detect changes occurring in the acute phase following injury nor to assess the primary impact of hemorrhage progression.

\section{CONCLUSION}

This study clearly demonstrates tICH expansion between the baseline CT scan and subsequent follow-up scans within $24 \mathrm{~h}$ in approximately half of the subjects, and that larger hematomas exhibit the greatest expansion. Furthermore, the earlier the initial CT scan is obtained after injury, the more likely it is that significant growth of the hematoma will be observed on subsequent scans, suggesting that most of the hematoma expansion occurs early after injury. This study also demonstrates the potential value of serial CT scans in the study of tICH evolution, and that CT scan measurements could serve as an early surrogate marker for the impact of therapeutic interventions with hemostatic agents.

\section{ACKNOWLEDGMENTS}

We wish to acknowledge Sheba Mathew, Thomas Henschel, and Patrick Rojas (Novo Nordisk Inc., Princeton, $\mathrm{NJ}$ ) for editorial and statistical assistance during the development of this manuscript, as well as Dr. Nikolai C. Brun (Novo Nordisk A/S, Bagsvaerd, Denmark) for his valuable input in the TBI clinical development program. This study was sponsored by Novo Nordisk A/S (Bagsvaerd, Denmark).

\section{AUTHOR DISCLOSURE STATEMENT}

Brett E. Skolnick, Ph.D. and Michael N. Tillinger, M.D., are employees of Novo Nordisk. All other authors were members of the steering committee and were compensated for their time by Novo Nordisk. Drs. Skolnick and Narayan own common shares of Novo Nordisk. All members of the Traumatic Intracerebral Hemorrhage Study Group were compensated by Novo Nordisk for their time in running this clinical trial at their institutions, as per normal practice.

\section{REFERENCES}

Broderick, J.P., Brott, T.G., Duldner, J.E., Tomsick, T., and Huster, G. (1993). Volume of intracerebral hemorrhage. A powerful and easy-to-use predictor of 30-day mortality. Stroke 24, 987-993.
Brott, T., Broderick, J., Kothari, R., et al. (1997). Early hemorrhage growth in patients with intracerebral hemorrhage. Stroke 28, 1-5.

Brown, F.D., Mullan, S., and Duda, E.E. (1978). Delayed traumatic intracerebral hematomas. Report of three cases. J. Neurosurg. 48, 1019-1022.

Bullock, R., Chesnut, R.M., Clifton, G., et al. (2000). Guidelines for the management and prognosis of severe head injury. Brain Trauma Foundation. J. Neurotrauma 17, 457-554.

Carrick, M.M., Tyroch, A.H., Youens, C.A., and Handley, T. (2005). Subsequent development of thrombocytopenia and coagulopathy in moderate and severe head injury: support for serial laboratory examination. J. Trauma 58, 725-729.

Chang, E.F., Meeker, M., and Holland, M.C. (2006). Acute traumatic intraparenchymal hemorrhage: risk factors for progression in the early post-injury period. Neurosurgery 58, 647-656.

Chieregato, A., Fainardi, E., Morselli-Labate, A.M., et al. (2005). Factors associated with neurological outcome and lesion progression in traumatic subarachnoid hemorrhage patients. Neurosurgery 56, 671-680.

Clifton, G.L., Grossman, R.G., Makela, M.E., Miner, M.E., Handel, S., and Sadhu, V. (1980). Neurological course and correlated computerized tomography findings after severe closed head injury. J. Neurosurg. 52, 611-624.

Diaz, F.G., Yock, D.H., Jr., Larson, D., and Rockswold, G.L. (1979). Early diagnosis of delayed posttraumatic intracerebral hematomas. J. Neurosurg. 50, 217-223.

Doppenberg, E.M., Choi, S.C., and Bullock, R. (2004). Clinical trials in traumatic brain injury: lessons for the future. J. Neurosurg. Anesthesiol. 16, 87-94.

Engstrom, M., Romner, B., Schalen, W., and Reinstrup, P. (2005). Thrombocytopenia predicts progressive hemorrhage after head trauma. J. Neurotrauma 22, 291-296.

Fainardi, E., Chieregato, A., Antonelli, V., Fagioli, L., and Servadei, F. (2004). Time course of CT evolution in traumatic subarachnoid haemorrhage: a study of 141 patients. Acta Neurochir. (Wien.) 146, 257-263.

Geerts, W.H., Pineo, G.F., Heit, J.A., et al. (2004). Prevention of venous thromboembolism: the Seventh ACCP Conference on Antithrombotic and Thrombolytic Therapy. Chest 126, 338S-400S.

Gudeman, S.K., Kishore, P.R., Miller, J.D., Girevendulis, A.K., Lipper, M.H., and Becker, D.P. (1979). The genesis and significance of delayed traumatic intracerebral hematoma. Neurosurgery 5, 309-313.

Kannus, P., Palvanen, M., and Niemi, S. (2001). Time trends in severe head injuries among elderly Finns. JAMA 286, 673-674.

Kothari, R.U., Brott, T., Broderick, J.P., et al. (1996). The ABCs of measuring intracerebral hemorrhage volumes. Stroke 27, 1304-1305. 


\section{HEMORRHAGE PROGRESSION IN TRAUMATIC BRAIN INJURY}

Kurtoglu, M., Yanar, H., Bilsel, Y., et al. (2004). Venous thromboembolism prophylaxis after head and spinal trauma: intermittent pneumatic compression devices versus low molecular weight heparin. World J. Surg. 28, 807-811.

Lobato, R.D., Gomez, P.A., Alday, R., et al. (1997). Sequential computerized tomography changes and related final outcome in severe head injury patients. Acta Neurochir. (Wien.) 139, 385-391.

Luukinen, H., Herala, M., Koski, K., Kivela, S.L., and Honkanen, R. (1999). Rapid increase of fall-related severe head injuries with age among older people: a population-based study. J Am. Geriatr. Soc. 47, 1451-1452.

Maas, A., Marmarou, A., and Murray, G. (2007). Prognosis and clinical trial design in Traumatic Brain Inury: The IMPACT study. J. Neurotrauma 24, 232-238.

Mayer, S.A., Brun, N.C., Begtrup, K., et al. (2005). Recombinant activated factor VII for acute intracerebral hemorrhage. N. Engl. J. Med. 352, 777-785.

Merino-deVillasante, J., and Taveras, J.M. (1976). Computerized tomography (CT) in acute head trauma. AJR Am. J. Roentgenol. 126, 765-778.

Narayan, R.K., Greenberg, R.P., Miller, J.D., et al. (1981). Improved confidence of outcome prediction in severe head injury. A comparative analysis of the clinical examination, multimodality evoked potentials, CT scanning, and intracranial pressure. J. Neurosurg. 54, 751-762.

Narayan, R.K., Kishore, P.R., Becker, D.P., et al. (1982). Intracranial pressure: to monitor or not to monitor? A review of our experience with severe head injury. J. Neurosurg. 56, 650-659.

Narayan, R.K., Michel, M.E., Ansell, B., et al. (2002). Clinical trials in head injury. J. Neurotrauma 19, 503-557.

Oertel, M., Kelly, D.F., McArthur, D., et al. (2002). Progressive hemorrhage after head trauma: predictors and consequences of the evolving injury. J. Neurosurg. 96, 109-116.

Pace, M.C., Cicciarella, G., Barbato, E., et al. (2006). Severe traumatic brain injury: management and prognosis. Minerva Anestesiol. 72, 235-242.

Procaccio, F., Stocchetti, N., Citerio, G., et al. (2000). Guidelines for the treatment of adults with severe head trauma (Part
I). Initial assessment; evaluation and pre-hospital treatment; current criteria for hospital admission; systemic and cerebral monitoring. J. Neurosurg. Sci. 44, 1-10.

Reilly, P.L., Graham, D.I., Adams, J.H., and Jennett, B. (1975). Patients with head injury who talk and die. Lancet 2, 375-377.

Servadei, F., Murray, G.D., Penny, K., et al. (2000). The value of the "worst" computed tomographic scan in clinical studies of moderate and severe head injury. European Brain Injury Consortium. Neurosurgery 46, 70-75.

Stein, S.C., and Smith, D.H. (2004). Coagulopathy in traumatic brain injury. Neurocrit. Care 1, 479-488.

Stein, S.C., Spettell, C., Young, G., and Ross, S.E. (1993). Delayed and progressive brain injury in closed-head trauma: radiological demonstration. Neurosurgery 32, 25-30.

Stein, S.C., Young, G.S., Talucci, R.C., Greenbaum, B.H., and Ross, S.E. (1992). Delayed brain injury after head trauma: significance of coagulopathy. Neurosurgery 30, $160-165$.

Yamaki, T., Hirakawa, K., Ueguchi, T., Tenjin, H., Kuboyama, T., and Nakagawa, Y. (1990). Chronological evaluation of acute traumatic intracerebral haematoma. Acta Neurochir. (Wien.) 103, 112-115.

Zimmerman, R.D., Maldjian, J.A., Brun, N.C., Horvath, B., and Skolnick, B.E. (2006). Radiologic estimation of hematoma volume in intracerebral hemorrhage trial by CT scan. AJNR Am. J. Neuroradiol. 27, 666-670.

Address reprint requests to: Raj K. Narayan, M.D. Mayfield Professor and Chairman Department of Neurosurgery University of Cincinnati

The Neuroscience Institute at University Hospital and The Mayfield Clinic 231 Albert Sabin Way Cincinnati, $\mathrm{OH}$ 45267-0515

E-mail: raj.narayan@uc.edu 